\title{
Stakes and Probabilities in Information Purchase ${ }^{1}$
}

\author{
Mrchael F. O'Connor, ${ }^{2}$ Cameron R. Peterson, and Terrance J. Palmer \\ University of Michigan
}

\begin{abstract}
Subjects purchased information to increase $P$ (probability of a correct decision) in tasks with net expected value held constant. In Experiment 1, Ss purchased more information, and thereby increased $P$, when stakes were higher. Experiment 2 showed a positive relation between stakes and $P$ regardless of which variable was independent. The hypothesis that Ss were holding variance constant could not account for this tradeoff, however, because $S_{\mathrm{s}}$ treated $P$ as relatively more important than stakes. Finally, under the assumption that risk increases with stakes and decreases with $P$, the riskier $S$ s treated $P$ as relatively more important than did the less risky $S$ s.
\end{abstract}

A decision maker can frequently increase the probability of making a correct decision by obtaining information pertaining to the states of nature relevant to that decision. However, such information is costly in terms of time, effort, or money. A class of models within statistical decision theory is devoted to the problem of the amount of information that should be purchased when costly information will increase the probability of a correct decision. These models provide a means for finding the optimal amount of information to purchase; the number of data that will maximize the net expected value of the decision.

Figure 1 shows how the expected value of a decision varies as the number of data purchased $N$ increases. Although the results of Fig. 1 are derived for a specific case, the forms of the functions are typical. In this case, the decision maker has the task of deciding whether a population proportion is 0.6 or 0.4 . The prior probability of each proportion is onehalf. The decision maker pays .0125 dollars for each datum sampled from the population, wins one dollar if his decision is correct, and loses one dollar if he is incorrect. The gross expected value is the expected value of the decision without regard to the cost of data. Since data sampled will in-

${ }^{1}$ Research conducted in the Engineering Psychology Laboratory, Institute of Science and Technology, University of Michigan. The research was supported by the Wood Kalb Foundation and by the U.S.P.H.S. Mathematical Psychology Training Grant No. 03077 to the University of Michigan.

${ }^{2}$ Requests for reprints should be sent to Michael F. O'Connor, Engineering Psychology Laboratory, Highway Safety Research Institute, Institute of Science and Technology, University of Michigan, Ann Arbor, Michigan 48105.

(C) 1972 by Academic Press, Inc. 


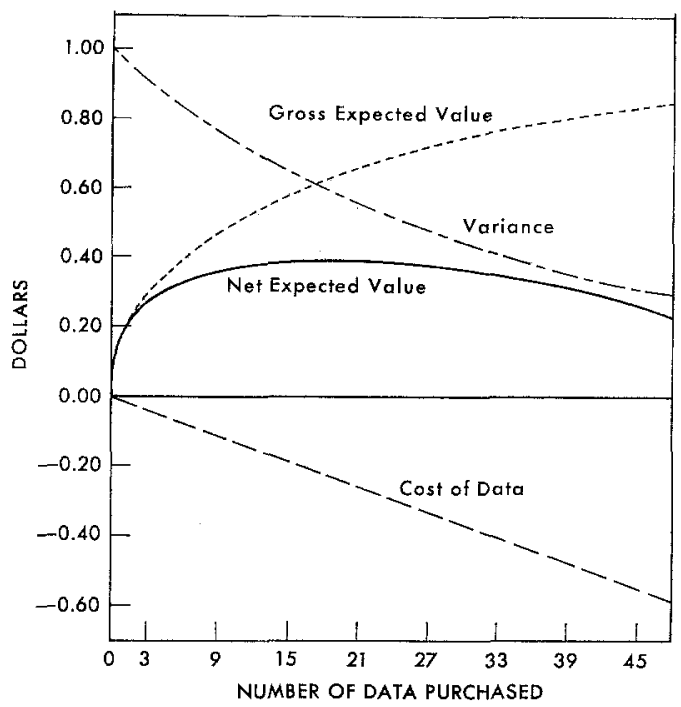

Fig. 1. Relations between gross expected value, net expected value, variance, and cost as a function of the number of data purchased.

crease the probability of being correct at a decreasing rate, the gross expected value of the decision increases rapidly at first and then asymptotes at one dollar. The cost function indicates the increase in the cost of data as a function of $N$. Since each datum costs a constant amount, this function is linear. The net expected value (EV) function is equal to the gross expected value minus the cost of the data. This function increases to a maximum and then decreases. It is optimal to purchase the number of data associated with the maximum of the net $\mathrm{EV}$ function.

An important characteristic of this function is that it is very flat in a broad region around its maximum. Moderate deviations from the optimal $N$ result in only a relatively small decrement in the net expected value of the decision. Schlaifer has pointed out that in a wide class of situations, ". . a moderate error in sample size is of no practical importance whatever-a sample which is 10 percent above or below optimum cannot increase expected total loss by as much as 6/10 of 1 percent" (Schlaifer, 1961, p. 337). This llatness in the region of the optimum is a general characteristic of information purchase tasks, so $\mathrm{EV}$ cannot be a very important determinant of how much information should be purchased within the general range of the optimum.

It is therefore important to isolate which variables, if any, determine $N$ in the region where net $\mathrm{EV}$ is relatively constant. One obvious candidate is stakes. Previous research has shown that subjects increase $N$ as stakes increase and when the cost of data is held constant (Peterson \& 
Beach, 1967). But in that case, EV is tied to stakes. Experiment 1 untied those two variables; the cost of data within each level of stakes was constructed so that $\mathrm{EV}$ was zero regardless of the number of data purchased.

\section{EXPERIMENT 1}

\section{Method}

Experimental design. The Ss purchased samples of data from a binomial population for the purpose of deciding whether the population proportion was .60 or .40 . Each $S$ won $\mathrm{X}$ dollars for a correct decision and lost $\mathrm{X}$ dollars for an incorrect decision. In order to vary dispersion over a wide range, $\mathrm{X}$ took on values of $.10, .25, .50,1.00,2.00$, and 5.00 . In order to hold $\mathrm{EV}$ constant at zero to the nearest cent, the cost of data increased as a negatively accelerated function of $N$. Specifically, the cost of any sample size was equal to the gross EV for that sample size. The $S$ s could purchase zero data or any odd number from 1 to 47 .

Apparatus. The $S$ faced a panel of two columns of lights. Each column contained 24 pairs of red and green bulbs, with a button between the members of each pair. Pushing the button for a particular $N$ caused the appropriate bulbs of the first $N$ pairs to light. For each pair, the color of the light represented the datum that was sampled randomly with replacement from the selected population. A plugboard behind the panel controlled which bulb in each of the 48 pairs would light. Forty-six different plugboards represented 23 independent samples and their mirror images. Thus, insertion of a different plugboard caused a different sequence of bulbs to light. Below the sample display were two decision buttons corresponding to the two different binomial populations. Pushing either decision button lit the bulb corresponding to the correct population proportion.

Procedure. The $E$ instructed $S$ about the nature of the informationpurchase task and the apparatus. He further indicated that the task was structured such that some $S$ s may win and others lose, but the average winnings should be about zero. The $E$ described the six different levels of stakes and pointed out a display of the cost function associated with each level. On each trial the $E$ selected a population proportion by inserting a randomly selected plug into the apparatus. The $S$ then purchased a sample of data by pushing the appropriate button. After observing the outcome of that sample he made his prediction of the population proportion by pushing the desired button and then received immediate feedback about whether he was right or wrong. The $E$ then informed the $S$ of tive current total of his wins or losses. 
Each block of six trials included all six levels of stakes presented in a random order. Because of the magnitude of the money involved, which included wins or losses of up to $\$ 5.00$, the range of the amounts that $S \mathrm{~s}$ could win or lose was too large to be acceptable for $S$ s from a college student population. This range was lowered by the following procedure, about which $S$ s were not informed. If any $S$ was winning more than $\$ 8.00$ he faced unfavorable sequences in which the undiagnostic plugs, which pointed least strongly to the correct population or even to the incorrect population, were systematically associated with high stakes. If, on the other hand, the $S$ was losing more than $\$ 2.00$ he faced a favorable sequence in which the diagnostic plugs were associated with the high stakes and the undiagnostic plugs with the low stakes. Otherwise, $S$ encountered a neutral sequence for which diagnosticity was not related to stakes. Most of the time Ss operated in the neutral sequence. A post-experimental interview was conducted to learn whether any of the $S \mathrm{~s}$ suspected that the process of selecting plugs was in any way not random. Two of the Ss indicated that they were suspicious of the procedure.

Each $S$ participated in eleven blocks of six trials unless he was too far ahead or behind with respect to his winnings. In that case he continued participating in the experiment until his winnings fell in the range of $\$ 0$ to $\$ 6.00$. Ss were not informed before the experiment about how many trials in which they would have to participate.

Subjects. Twenty-four men students at the University of Michigan served as $S \mathrm{~s}$ individually. They were paid $\$ 1.50$ an hour plus their winnings in the experiment. The $S_{\mathrm{s}}$ volunteered with the knowledge that it was possible to lose some of their own money in the experiment. A large proportion of the $S_{s}$ who were initially contacted to participate in the experiment refused because of the possibility of losing their own money. This selection factor may have led to $S$ s with higher than average levels of preferred risk. Ten of the $S$ s were graduate students and fourteen were undergraduates.

\section{RESULTS}

The results displayed in Fig. 2 indicate that $N$ inereases with the level of stakes. These results were obtained by first calculating the mean $N$ purchased by each $S$ within each level of stakes. Means and their standard errors were then calculated across $S \mathrm{~s}$ and are displayed in Fig. 2. The first four blocks of trials were a priori designated as practice trials and are thus omitted from the data analysis. The results displayed are based on the remaining complete blocks of trials. The number of blocks analyzed ranged from seven to twelve for the different Ss.

Figure 2 shows that the mean $N$ increased almost linearly as a function 


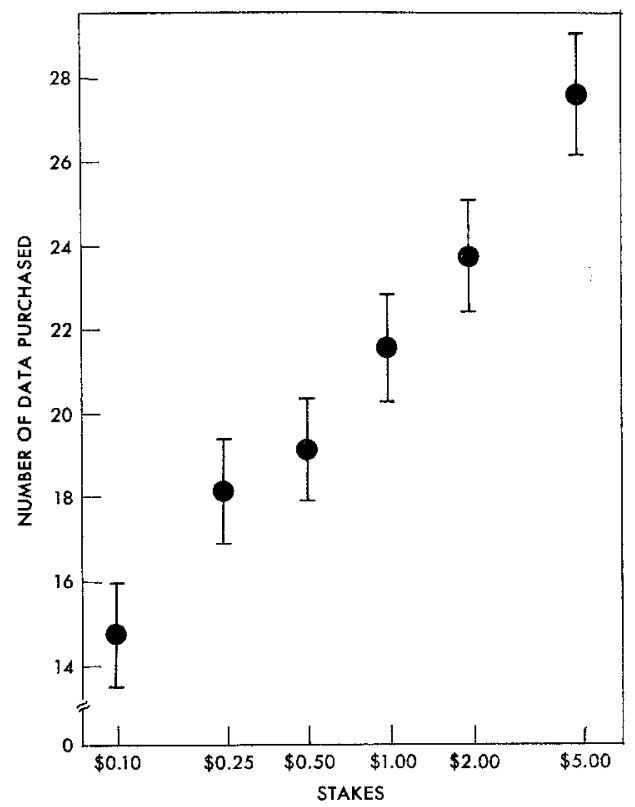

FIG. 2. Means and standard deviations as a function of the logarithm of the stakes.

of the $\log$ of the stakes. This linear relation held for most of the individual Ss. The median correlation between $N$ and $\log$ stakes was .87 ; nine of the $S$ s had correlations of more than .90 and only three were negative. The two $S \mathrm{~s}$ who indicated that they were suspicious of the experimental procedure are two of the three with negative correlations. The correlation between $\log$ stakes and $N$ was higher than that between stakes and $N$ for 17 of 24 Ss.

\section{DISCUSSION}

The results of Experiment 1 show that most $S$ s increase $N$ in response to increased stakes, even when $\mathrm{EV}$ is held constant. The direction of this effect is consistent with the hypothesis that each person strives to maintain the variance of his decisions at a relatively constant level. Several experiments of the choice-among-bets type have investigated the specific hypothesis of variance preference (e.g., Coombs \& Pruitt, 1960; Lichtenstein, 1967; Slovic, Lichtenstein, \& Edwards, 1965). Some of the results have been positive, but the evidence is generally inconclusive.

In a situation where $S$ wins $A$ dollars with probability $P$ and otherwise wins $B$ dollars, the variance of the decision is $P(1-P)(A-B)^{2}$. Figure 1 illustrates how the variance decreases with $N$ when stakes are constant 
in that situation. The hypothesis that $S$ s maintain an ideal level of variance implies that any change in variance away from that ideal caused by a change in stakes will lead $S$ to compensate by a corresponding change in $P$ so as to bring variance back to his preferred level. This requires the directional tradeoff between $P$ and stakes found in Experiment 1 . However, the tradeoff that occurred was not large enough. Subjects did not increase $N$ (and thus $P$ ) as much in response to changed stakes as would Ss holding variance constant.

There are at least two reasons why an $S$ who was attempting to hold variance constant would fail to increase $N$ sufficiently in response to increased stakes. One reason is that he might not know the appropriate relation between $N$ and $P$. Even if he understands that $P$ increases monotonically with $N$, he may assume that the increase is steeper than it actually is. Further, Ss may have been only partially sensitive to manipulations of the independent variable. Several experiments on decision making have demonstrated such partial sensitivity on the part of $S_{\mathrm{s}}$ (Peterson \& Beach, 1967). Either of these reasons could account for the failure of Experiment 1 to support the variance-preference hypothesis. Therefore, Experiment 2 was designed to increase the transparency of $P$ and to measure the amount of partial sensitivity to changes in stakes.

\section{EXPERIMENT 2}

In an effort to make the probability of winning more transparent, Experiment 2 required $S$ to purchase a single datum at his selected bag composition rather than to determine $P$ by deciding how many data to purchase. Thus, for example, $S$ could select a $P$ of $90 \%$ by purchasing a single datum sampled from a population characterized by $90 \%$ blue chips and $10 \%$ red chips. The cost of the sample increased with $P$ such that $\mathrm{EV}$ was constant at zero.

A switching of independent with dependent variables served to control for the problem of partial sensitivity. The $E$ set the level of stakes on even-numbered trials and the Ss selected the level of diagnosticity within which to purchase his information. On odd numbered trials the $E$ specified the level of diagnosticity and the $S$ responded by selecting a level of stakes at which he wished to play the game. In order to make it possible for $S$ to hold variance at a constant level, the diagnostic values ranged from a $P$ of 0.5 to a $P$ of 1.0. The cost of information equalled the gross $\mathrm{EV}$ making the net $\mathrm{EV}$ of any game zero.

\section{METHOD}

Experimental design. The experiment was divided into a practice session with imaginary stakes, and another session played for real money. 
During both sessions $E$ determined either $P$ or stakes and $S$ responded with stakes or $P$, respectively. The $E$ then drew a poker chip from a bookbag with the predominant proportion equal to $P$, and $S$ decided which color of chip was predominant, red or blue. The remaining procedure was similar to Experiment 1.

Apparatus. Each of the four bookbags used in practice sessions contained fifty poker chips with either red or blue predominant. The proportion of predominant chips $P$ was either .60 or .70 . Other apparatus included displays of the population proportions, an adding machine to keep track of wins and losses, and answer sheets on which each $S$ recorded his decisions.

Procedures. The $E$ acquainted $S$ with the nature of the game by means of 68 practice trials with imaginary money. During the practice trials, $E$ specified either $P$ or stakes and $S$ responded with his desired stake or $P$. During the trials with real money, $E$ specified each $P$ and each stake at least once again in random order. Stakes took on values of $\$ .10, \$ .25$, $\$ .50, \$ 1.00, \$ 2.00$, and $\$ 5.00$. The $P$ took on the values $.50, .60, .70, .80, .85$, $.90, .93, .96, .98, .99$, and 1.0. There were many $P$ 's in the range of .90 to 1.0 because large changes in variance take place in this range as compared to, for example, the $.50-.60$ range. The actual sampling process had been completed by $E$ prior to the experiment by sampling from the appropriate binomial population. This was explained to each $S$ and no one objected or indicated suspicion. As in Experiment 1 it was possible for Ss to get either too far ahead or behind. Accordingly, neutral, favorable, and unfavorable sequences were used in a manner similar to that of Experiment 1. Each $S$ played until his winnings were in the acceptable range. The number of real-money trials varied from a necessary minimum of 17 to a maximum of 40 .

Subjects. Twenty-five men students at the University of Michigan served as $S_{\text {s. }}$. They responded to invitations sent from a subject pool. Each $S$ was informed of the possibility of losing some of his own money.

\section{RESULTS}

Experiment 2 tested the hypothesis that subjects strive to maintain an ideal or fixed level of variance. If that level is $K$, then

$$
P(1-P)(A-B)^{2}=K \text {. }
$$

By taking logs and transposing variables, Eq. 2 specifies the following linear relation between probabilities and stakes

$$
\log (P(1-P))=\log K-2(\log (A-B)) .
$$

Note that $P(1-P)$ is a maximum when $P=.5$ and decreases as $P$ ap- 
proaches one or zero. Equation 2 implies that each unit increase in log $(A-B)$ will be compensated for by a corresponding two-unit decrement in $\log (P(1-P))$. Thus, the constant variance hypothesis implies a negative correlation between $\log (P(1-P))$ and $\log (A-B)$ for each $S$; the best fitting regression line should have a slope of -2.0 .

Of the $24 \mathrm{Ss}$ who completed the experiment (one refused to play for real money), 21 exhibited negative correlations, eleven of which were more extreme than -.85 . Of the Ss without extreme negative correlations, the most risky $S$ always chose a $P$ of .5 or stakes of $\$ 5.00$; two $S$ s displayed positive correlations; and others rather consistently chose some constant intermediate level of probability or stakes.

The $11 \mathrm{Ss}$ characterized by extreme negative correlations yielded an interesting pattern of results. Contrary to the partial sensitivity found in previous experiments, the slope of the regression line was essentially independent of whether $P$ or stakes was the dependent variable. The correlation between regression slopes in the two conditions was .87. Slopes were slightly more negative when $P$ was independent; the mean difference was only -.10 . The regression slopes ranged between -.13 and -.84 , substantially flatter than the -2.0 predicted by the constant variance model. As compared with that model, Ss treated probability of winning as relatively more important than stakes.

Not only were the slopes flatter than prescribed by the constant variance model, but the degree of flatness depended upon both the mean $\log (A-B)$ and the mean $\log P(1-P)$. The correlation of slope with the mean $\log (A-B)$ was .90 when stakes comprised the dependent variable; the correlation of slope with mean $\log P(1-P)$ was .85 when $P$ was the dependent variable. Thus, Ss who chose high $\log (A-B)$ or high $\log$ $P(1-P)$ tended to have flatter slopes.

\section{DISCUSSION}

As expected, most $S$ s compensated for increases in stakes by paying relatively more for information by purchasing either more data or a more diagnostic datum. One interpretation of this behavior is that there is a tendency to maintain risk at some constant, intermediate level. There is little agreement in the literature about what comprises risk, but we are willing to assume that it increases with stakes and according as the probability of a correct terminal decision decreases. If so, when the experimenter changed risk by manipulating an independent variable the Ss responded by changing risk in the opposite direction on the dependent variable.

Previous investigators have proposed variance as a measure of risk 
and also that each person has a variance preference, i.e., he prefers to maintain variance at some fixed level. However, the present results suggest that, even though the components of variance, $P(1-P)$ and $A-B$, may contribute to risk, $S_{\text {s }}$ may combine them in some way other than specified by the variance. That is, Ss may tend toward holding risk constant, but may perceive risk as some measure of dispersion other than variance. This interpretation is strengthened by results of previous research in which some model other than the mean square deviation accounts for $S s^{\prime}$ estimates of relative variability (Beach \& Scopp, 1968; Hofstatter as reported in Brunswik, 1956). The best-fitting exponent of the deviations is typically less than 2 ; in fact, it is usually less than $\mathbf{1}$. In contrast to the interpretation of the variance measure, estimates of perceived variability tend to be more sensitive to differences in variability that are near rather than far from the mean or expected value. We suspect that it might be possible to account for some of the present results by assuming that $S$ s are attempting to hold some other measure of variability constant, but we have not been able to derive any simple relation between the best-fitting exponent applied to deviations from EV and the slope relating $\log P(1-P)$ to $\log (A-B)$. Our intuition is that the slope should become flatter as the deviation exponent decreases.

Whatever the relation between dispersion preferences and information purchase, $S \mathrm{~s}$ in the present experiments made tradeoffs that treated probability as relatively more important than stakes. And the control condition in Experiment 2, which switched independent and dependent variables, indicates that this relation cannot be accounted for by a partial sensitivity on the part of $S_{s}$. The slope of the regression line was essentially the same, regardless of whether stakes or probability was treated as the independent variable. But more interesting than the general nature of the tradeoff is the fact that it depended upon S's propensity for taking risks. It seems congruent with common sense that the riskier $S$, who tended to choose higher average stakes and smaller average probabilities of a correct decision, was characterized by a flatter slope, i.e., tended to deemphasize stakes relative to probability. This behavior is consistent with the viewpoint that it is the conservative rather than the risky person who focusses on stakes. He prefers not to gamble and is willing to purchase an insurance policy in order to avoid a small chance of a large loss. He will pay in terms of the net expected value in order to reduce his perceived level of risk. This seems to be a reasonable interpretation of why one person would be willing to purchase more information than another person, especially in the range where the $\mathrm{EV}$ function is flat. 


\section{REEERENCES}

BEACH, L. R. \& ScOPP, T. S. Intuitive inferences about variances. Organizational Behavior and Human Performance, 1968, 3, 109-123.

Brunswik, E. Perception and the representative design of experiments. Berkeley: University of California Press, 1956.

Cоомвs, C. H. \& Pruixt, D. G. Components of risk in decision making: Probability and variance preferences. Journal of Experimental Psychology, 1960, 60, 265-277.

Lichtenstein, S. C. Bases for preferences among three-outcome bets. Journal of Experimental Psychology, 1967, 69, 162-169.

Peterson, C. R. \& Beach, L. R. Man as an intuitive statistician. Psychological Bulletin, 1967, 68, (1), 29-46.

SCHLAIFER, R. Introduction to statistics for business decisions. New York: McGrawHill, 1961.

Slovic, P., Lichtenstein, S. C., \& Edwards, W. Boredom induced changes in preferences among bets. American Journal of Psychology, 1965, 78, 208-217.

RECeIVED: December 21, 1970 